\title{
PARALLEL SEED SELECTION METHOD FOR OVERLAPPING COMMUNITY DETECTION IN SOCIAL NETWORK
}

\author{
BELFIN R.V.\& E. GRACE MARY KANAGA*
}

\begin{abstract}
Social network analysis is one of the key areas of research during modern times. The social network is growing with more users and the ties between them day by day. This reason brings out many research queries and new conclusions from this area. Overlapping community detection in the social network is one such research problem which has acquired interest among researchers nowadays. Earlier, the investigation was in finding out algorithms to detect communities in the network sequentially. There are many distinguished findings toward overlapping community detection. Due to the velocity of data in the current era, the available algorithms will be a bit sluggish in processing the data. The proposed algorithm uses parallel processing engine to resolve this delay problem in the current scenario. The algorithm in parallel finds out the superior seed set in the network and expands it in parallel to find out the community. The work shows amazing improvement in the runtime and also detects quality groups in the network.
\end{abstract}

Key words: Overlapping community detection, Seed selection, Graph parallel processing, seed expansion

AMS subject classifications. 65Y05, 68R10, 91D30

1. Introduction. Complex networks are commonly utilized for modeling the synergies in real-world systems in various areas, such as sociology, biology, knowledge dispersion and many different fields. One important feature of this complex network is that the nodes are tightly connected with each other in groups and in turn, the groups will be loosely connected to each other. The tightly connected groups are called as communities. The communities usually have common properties. Therefore, finding the communities from a complex network could give many insights about the network. The community detection can be done in two different ways: Firstly, the network partition can be calculated to cut the graph into partitions. Secondly, the selection of seed nodes will be done and the local communities will be centered around these seeds.

The proposed work is of the latter category where, the most important nodes in the community will be identified using a parallel superior seed set selection $(\mathrm{P} 4 \mathrm{~S})$ algorithm. The identified superior seeds will be expanded by their neighborhood till it reaches the next seed. Since the algorithm expands using the neighborhood, it will form the closely knitted group around the seed nodes.

Seed selection process is an important process in the field of network science. Usually, the measures for calculating the important nodes from the target network is named as centrality measures [24, 25]. There are many centrality measures, to name a few, Degree [31], Betweenness [34], Closeness [18], Eigen vector [35], Page Rank [32] and so on. These seed nodes play an important role in finding a good community in community detection problem and fast spreading in information diffusion application.

2. Related Works. Data nowadays is huge and need to be processed as fast as possible. There is a need of parallel algorithms to process the volume of data which comes in high velocity. Processing the data in the faster may fetch the organizations a good profit. There are lots of seed selection algorithms available for different applications. Each application might have to adopt different seed selection algorithms which match the application requirement. For example, if the application is a marketing application, the out-degree centrality or the page rank centrality may be used for the seed selection process. So, each centrality measures[9, 10, 2] will have their own limitations with various applications. The proposed method finds out a generic seed by combining various centralities.

There are some related works available in the literature. There are some diffusion models [40, 28, 21, 7] and community detection algorithms $[36,3,26,14,11]$ which will have a seed selection part in their model $[22,12,27,6]$. Some of the algorithms selects the seeds in random and optimize its result at the end of the process [15]. There is a excellent study on seed nodes in [13]. Evolutionary algorithms are used to find out the seed nodes in some cases [34].

*Department of Computer Science and Engineering, Karunya institute of technology and sciences, Coimbatore, Tamilnadu, India. (belfin@karunya.edu, grace@karunya.edu). 
There are several graph computations algorithm available for large-scale graphs, various parallel modules have been developed, e.g., Pregel [20], GraphLab [19] Giraph++ [8], GraphX [37], GRACE [30], GPS [23] and Blogel [38], based on MapReduce [5] and BSP (Bulk Synchronous Parallel) models [29].

The proposed algorithm uses GraphX to process the graph in parallel. Our previous work Superior seed set selection algorithm (4S) [1] was extended to parallel processing in this article. The experiment result proves that the proposed parallel algorithm can find out the good clusters fastest then the available algorithms.

3. Problem Statement. Some of the seed selection algorithms which are already available are using random nodes for selecting the seed node. The selection of a seed node or a seed set is really important in the algorithms which use seeds to find out communities. Since, the community detection algorithm needs to work in unsupervised way the seeds should be excellent to obtain a perfect local community. The selection of excellent seeds will reduce the number iterations in the community detection algorithm.

Nowadays the data need to be processed is large because of the growing use of internet and social media. Reducing iterations will not be enough to make the algorithm work faster. There is a need of parallel algorithms which can process the data in a synchronized manner. Parallel processing algorithms are modern nowadays. The uses of these kinds of algorithms will be fast and effective.

4. Problem Formulation. Assuming an undirected graph $G=(V, E)$. The nodes of the graph $G$ be $n=|V|$ nodes and the edges of the graph $G$ be $m=|E|$. The overlapping community detection algorithm is used to determine the community $C=C_{1}, \ldots, C_{x}$ of all the nodes of $G$. $|C|$ be the number of communities identified. In the proposed work, $|C|$ is the number of seed node selected from parallel superior seed set selection algorithm.

Various centrality measures $\mu_{i}$ for a node $i$ can be calculated which will be the importance measure of node $i$. Let us assume the graph to be undirected. The traditional methods uses any one of the centrality measures with respect to the use case, to define its seed set $S(G)=s_{i}, s_{j}, \ldots, s_{\kappa}$ where $s_{i}, s_{j}, \ldots, s_{\kappa} \in V$. Some models pick seed set randomly and gets the parameter $\kappa$ as input, to decide the number of seeds in the set. The difficulty in the conventional methods is that, the seed set need to be adjusted according to the circumstances of the problem. The proposed parallel, unified model determines the superior seed set $S(G)$ from the centrality measures collectively. This article introduces a threshold value $\tau$, which limits the number of seed nodes selected for the $S(G)$.

A community may be basically described as a collection of nodes that may share common features, or engage in similar roles in the network. Also, it is tightly knitted groups with a high density of inter community ties and a low density of intra-community ties. The proposed algorithm produces results that are composed of one of two types of assignments, crisp assignment of nodes or fuzzy assignment of nodes. With crisp assignment, the relationship between a node and a cluster is binary. That is, a node $i$ either belongs to community $C$ or does not. With fuzzy assignment, each node is associated with two or more communities. Throughout the article, the terms set, cluster, and community are used interchangeably.

5. Proposed work. The main idea of this work is to use the parallel processing architecture to find out the best seeds and subsequently the best seeds from $\mathrm{P} 4 \mathrm{~S}$ algorithm will be used to find out the communities hidden in the network. The work has been done with GraphX a module in spark parallel processing engine. The algorithms for the Parallel seed selection and parallel community detection has been explained below in the following sections. The parallel community detection framework is depicted in the Fig 5.1. The input of the algorithm will be the unlabeled network, for example, social graph or collaboration networks or the network of web pages. The output of the work will be the expanded communities from the selected seeds. The goodness of the communities is tested by comparing the inter-density and intra-density of the communities along with the graph density. The algorithm for the P4S has been given in the Algorithm 1. Algorithm 2 shows the parallel seed set expansion algorithm which finds out the communities.

GraphX is the Apache Spark ingredient for graph-parallel calculations, developed upon a division of mathematics called graph theory. It is a distributed graph processing framework that lies on top of the Spark core. GraphX inherits the Spark RDD with a Resilient Distributed Property Graph. The property graph is a directed multi-graph. The multiple edges it has will be parallel. The parallel edges permit multiple relationships between the same vertices. The Fig. 5.2 shows the architecture of the parallel $4 \mathrm{~S}$ and parallel community detection implemented in the spark. 


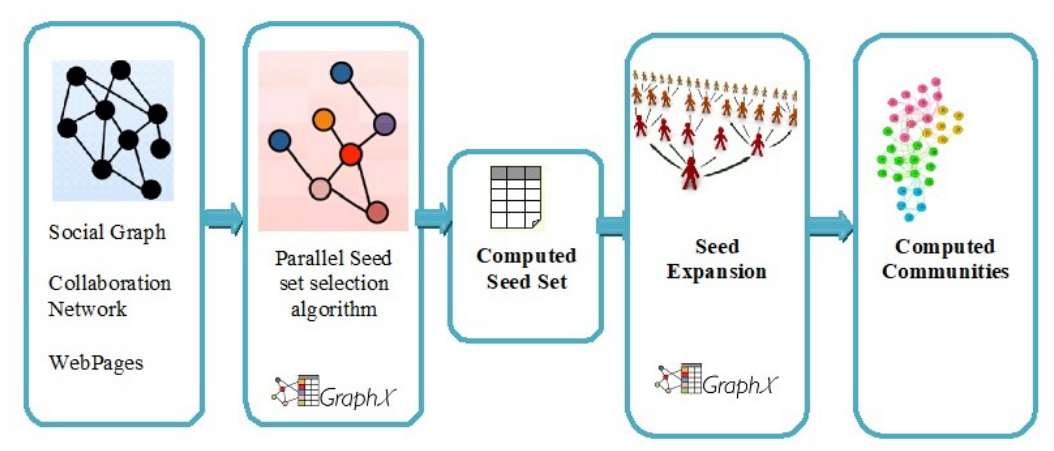

FIG. 5.1. Parallel community detection framework
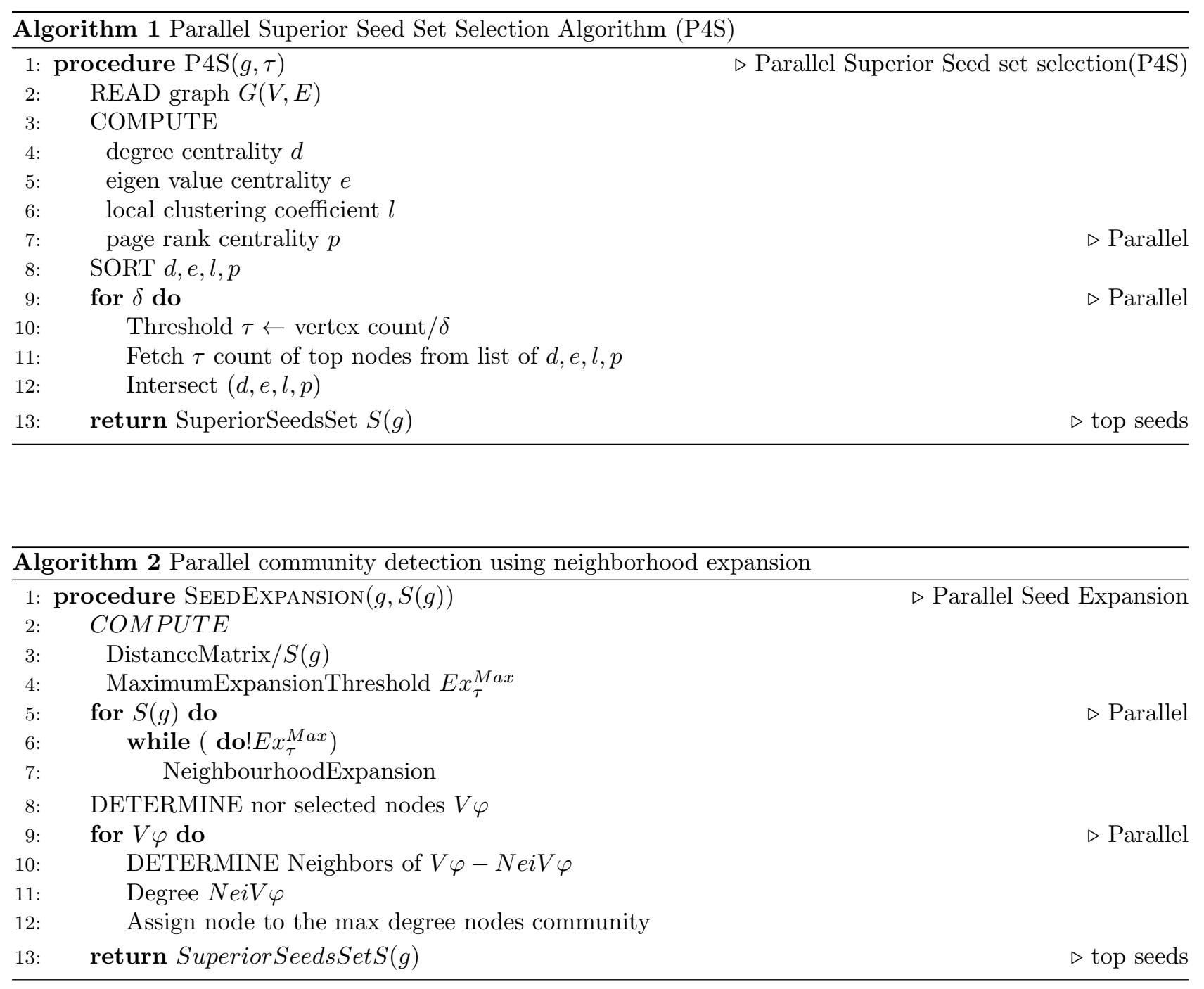


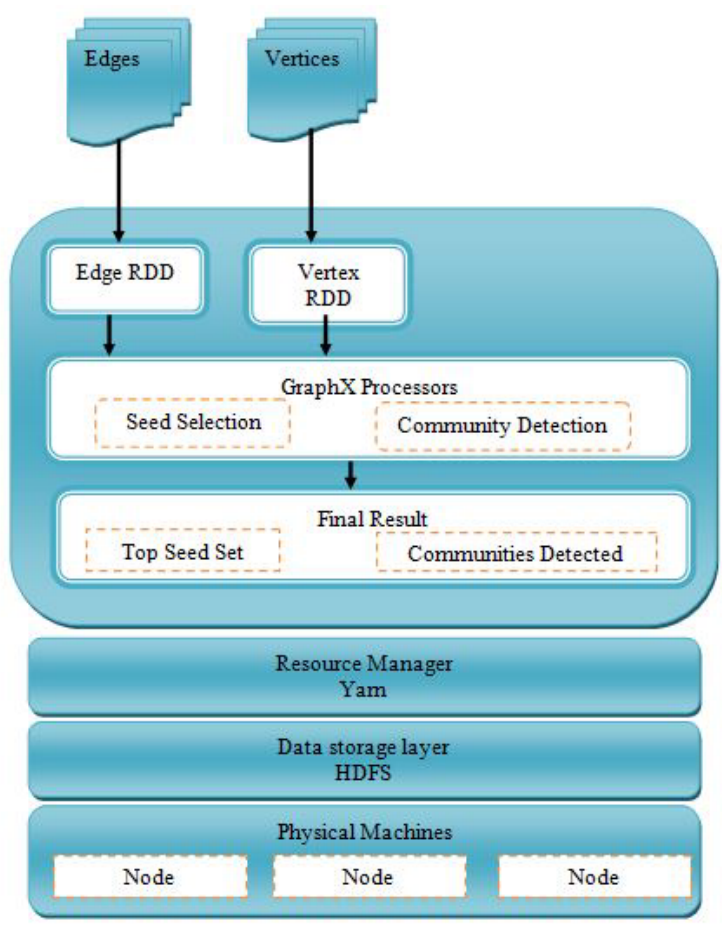

FIG. 5.2. Implementation of Parallel $4 S$ and Parallel community detection algorithm in Spark architecture

The GraphX processors will be used to compute the superior seed set and the seed expansion of the node to form community in parallel. Three node clusters was used for computing the communities in parallel.

5.1. Parallel Superior Seed Set Selection (P4S). Parallel superior seed set selection algorithm extracts the very important nodes in the input graph. The input data will be stored in the distributed file system for further processing. Centrality measures are used to coin out the important nodes in the network. Each centrality measure will have its own importance and use cases to work on. Combining these centrality measures can identify good seeds across all the centrality measures. For the experiment done for this work Page rank, Degree, Eigen value, Local clustering coefficient centralities was used. The centralities measure calculation will automatically run in parallel when GraphX is used. Sorting according to the centrality ranks will be done for all centrality measures used. A threshold value will be used to split the top ranks for all measures. Finally, set intersection of the top nodes from each centrality measure will be done to get the superior seed set. Fig. 5.3 depicts the process of finding the superior seed set.

5.2. Parallel Community Detection. Community detection from seed by expanding greedily through neighbors is a classical process. The proposed work is the modified version of the seed expansion in a parallel way. Since we have multiple seeds the algorithm will expand in parallel from the seeds till it reaches the next seed and stops expanding. The distance between the seeds will be calculated by the distance matrix. The $E x_{\tau}^{M a x}$ defined in the Algorithm 2 is the maximum expansion limit. The nodes which are not selected in the first iteration will be picked and added to its neighbors community. In case of more than one neighbor the highest degree node will add the ungrouped node to its community.

6. Experiment. The real-world datasets used for the experiments are from [17]. All the datasets are connected, undirected graphs. The datasets are from various categories like collaboration networks and product networks. The detailed information about the datasets is given in the Table 6.1.

Collaboration networks. In a collaboration network, vertices denote authors, and edges denote coauthorship. If authors $u$ and $v$ are co-authors, they will be connected by an edge. So, if an article is written 


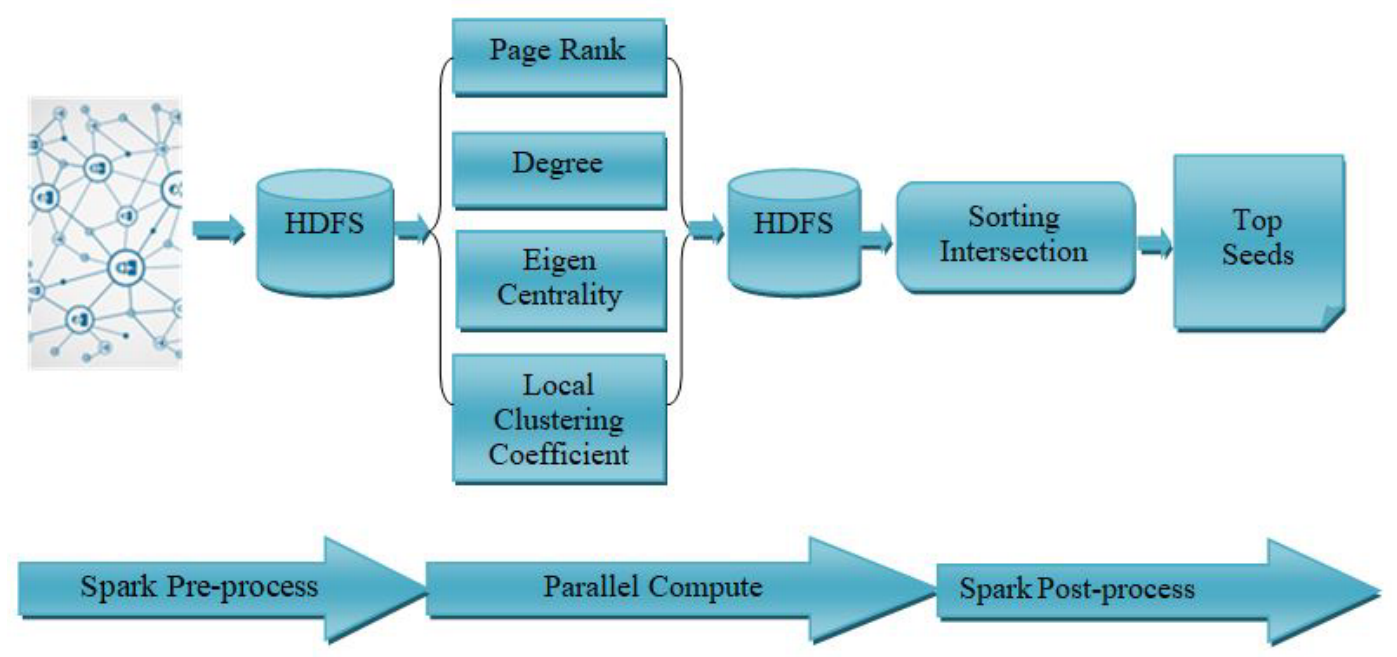

FIG. 5.3. Parallel $4 S$ algorithm for finding top seed set

TABLE 6.1

Summary of real-world networks used

\begin{tabular}{|l|l|l|l|l|l|l|}
\hline Graph & $\begin{array}{l}\text { No. of } \\
\text { Vertices }\end{array}$ & $\begin{array}{l}\text { No. of } \\
\text { edges }\end{array}$ & $\begin{array}{l}\text { Max. } \\
\text { Deg }\end{array}$ & $\begin{array}{l}\text { Avg. } \\
\text { Deg }\end{array}$ & Avg. CC & $\begin{array}{l}\text { Ground- } \\
\text { truth }\end{array}$ \\
\hline HepPh & 11,204 & 117,619 & 491 & 21.00 & 0.6216 & N/A \\
\hline AstroPh & 17,903 & 196,972 & 504 & 22.00 & 0.6328 & N/A \\
\hline CondMat & 21,363 & 91,286 & 279 & 8.50 & 0.6417 & N/A \\
\hline DBLP & 317,080 & $1,049,866$ & 343 & 6.60 & 0.6324 & Yes \\
\hline Amazon & 334,863 & 925,872 & 549 & 5.50 & 0.3967 & Yes \\
\hline Orkut & 731,332 & $21,992,171$ & 6933 & 60.10 & 0.2468 & Yes \\
\hline
\end{tabular}

by $\mathrm{n}$ authors, then their relationship will be represented as a clique in the network. HepPh, AstroPh, and CondMat networks are formed based on the journal submitted to High Energy Physics (Phenomenology) group, Astrophysics group, and Condensed Matter Physics group under the arXiv e-print service, respectively. The DBLP network is formed based on the DBLP computer science bibliography website.

Product network. In the Amazon product network, vertices denote products and edges denote copurchasing information. If products $u$ and $v$ are frequently co-purchased, then there will be an undirected edge between them.

Social networks. In a social network, vertices denote users of the social network and the edges denote social communications between them. Users can construct a friendship relationship with each other in this web application.

\section{Community Evaluation.}

7.1. Intra-Density, Inter-Density and Graph Density. The cohesiveness of the edges in a graph G can be readily attained by calculating the graph density $\rho$.

$$
\rho=\frac{|E|}{n(n-1) / 2}
$$

where $n$ is the count of nodes in the network and $n(n-1) / 2$ is the maximum possible edges and $|E|$ is the number of edges in graph $G$. 
Consider $c$ is a community in the given network $\mathrm{G}$ where $|G|=n$ and $|c|=n c$. Internal edges are edges which have it both sides situated inside the community $c$ and the external edges of community $c$ refer to the edges which connect a vertex in $c$ to the rest of the graph. The internal degree of vertex $v$ in community $c$ is denoted by $k_{v}^{i n t}$ is the number of edges connecting $v$ to other vertices in $c$ and the external degree of $v$ denoted as $k_{v}^{e x t}$ is the number of edges connecting $v$ to the rest of the graph. The intra cluster density $\delta^{i}(c)$ of a community $c$ is the ratio between the number of internal edges of $c$ and the number of all possible internal edges:

$$
\delta^{i}(C)=\frac{\left|E^{i}\right|}{n_{c}\left(n_{c}-1\right) / 2}
$$

where $\left|E^{i}\right|$ is the count of internal edges in the community.

Similarly, the inter-cluster density $\delta^{e}(c)$ is the ratio between the number of inter-cluster edges of $c$ and the number of all possible inter-cluster edges:

$$
\delta^{e}(C)=\frac{\left|E^{e}\right|}{n_{c}\left(n-n_{c}\right)}
$$

where $\left|E^{e}\right|$ is the count of inter community edges in the community.

The proposed parallel seed set selection algorithm has been implemented in the real-world datasets and has given a better result. The goodness of the community has been tested with the inter-cluster and intra-cluster density. Usually For overlapping communities, internal and external metric values will be used. Because, combination metrics and modularity scores will result in confusing values that should be inconsistent[15]. The goodness of the community using graph density $\rho$, intra-cluster density $\delta^{i}(c)$ and inter cluster density $\delta^{e}(c)$ can be given as:

$$
\delta^{i}(C)>\rho>\delta^{e}(C)
$$

The comparison of inter-cluster density, intra-cluster density and graph density of the real datasets are plotted in the result and discussion section. The experiment was done for the seed sizes $71,84,114,128,152$, 172, 207, 240 and 270.

7.2. Clustering Coefficient. A clustering coefficient is a measure of the degree to which nodes in a graph are inclined to tie together. Research results suggests that in most real-world networks, and in some social networks, nodes are likely to create tightly connected groups characterized by a pretty high density of ties; this likelihood will greater than the average probability of a connection randomly established between two nodes [33].

The local clustering coefficient $l_{i}$ for a vertex $i$ is then given by the ratio of links between the nodes within its neighbors divided by the number of edges possible between them. The local clustering coefficient for an undirected graph is given as:

$$
C_{i}=\frac{2\left|e_{j, n e i}: v_{j}, v_{n e i} \in N_{i}, e_{j, n e i} \in E\right|}{n e i_{i}\left(n e i_{i}-1\right) / 2}
$$

where vertex $v_{j}$, has $n e i_{i}$ neighbors, $\frac{n e i_{i}\left(n e i_{i}-1\right)}{2}$ edges will be the maximum possible edges exist among the vertices within the neighborhood.

8. Result and discussion. The problem of community assessment is still an open and difficult problem in spite of huge sum of work addressing this topic [13]. The experiment done on the real-world datasets are given on the summary Table 6.1. The values of inter-density, intra-density and graph density are compared to find the goodness of the cluster. The result from the six real-world datasets specified in Table 6.1 has been plotted in the Fig 8.1. Every dataset used for the experiment passes the test and it goes hand in hand with the Eq. (7.4). In all cases the intra-cluster density $\delta^{i}(c)$ from Eq. (7.2) of all the communities identified is greater than the graph density $\rho$ and the inter-cluster density $\delta^{e}(c)$ from Eq. 7.3 of nodes after finding communities is lesser then the graph density $\rho$ from Eq. 7.1. 

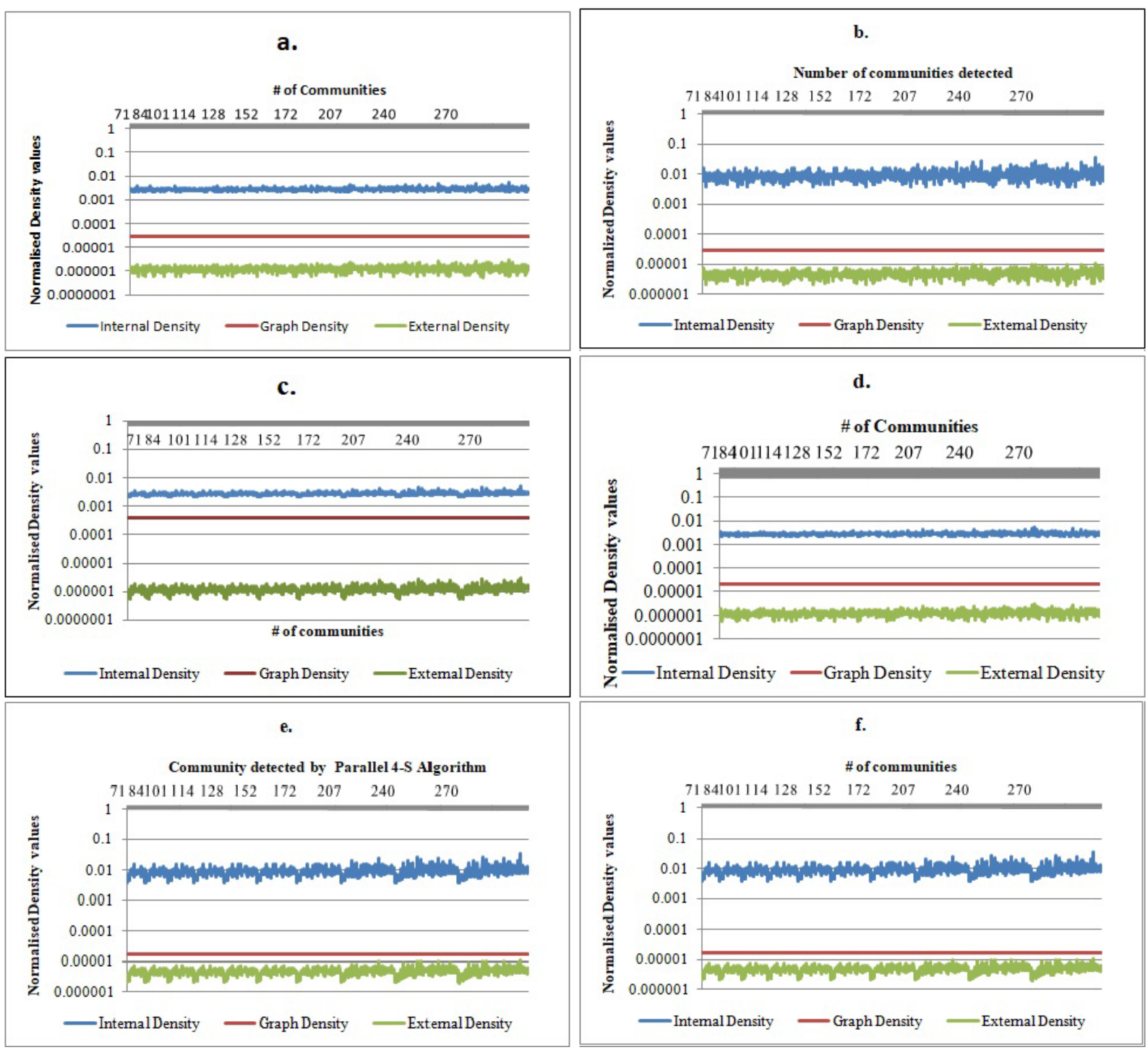

FIG. 8.1. Data comparison between the inter-cluster density, intra-cluster density and graph density: (a) Amazon dataset (b) AstroPh (c) CondMat (d) DBLP (e) HepPh (f) Orkut

The clustering coefficient can be a measure to find out the closely connected groups. The clustering coefficient equation is given the Eq. 7.5. The comparisons of clustering coefficient with the number of communities are given in the Fig. 8.2. The clustering coefficient of two datasets namely Amazon Fig. 8.2a and AstroPh Fig. $8.2 \mathrm{~b}$ are shown. The result shows an increasing trend in the clustering coefficient as the number of seeds increases. This shows that the larger the seed set higher the density of the community. Fig. 8.3 shows the comparison of clustering coefficient and number of nodes in each community. The result of comparison of average clustering coefficient of the entire network and the average community clustering coefficient is compared in the Fig. 8.4a and the comparison of average degree of the entire graph and the average degree of all the communities detected are shown as a bar plot in Fig. 8.4b. The plots clearly show that the density of the sub graphs increases after the community detection.

The running time factor is one of the important problems in community detection algorithms. When the input graph becomes larger the operations in graph will become complex and operations on it will be costly. The running time of the algorithm was compared with five important algorithms from the literature shown in 


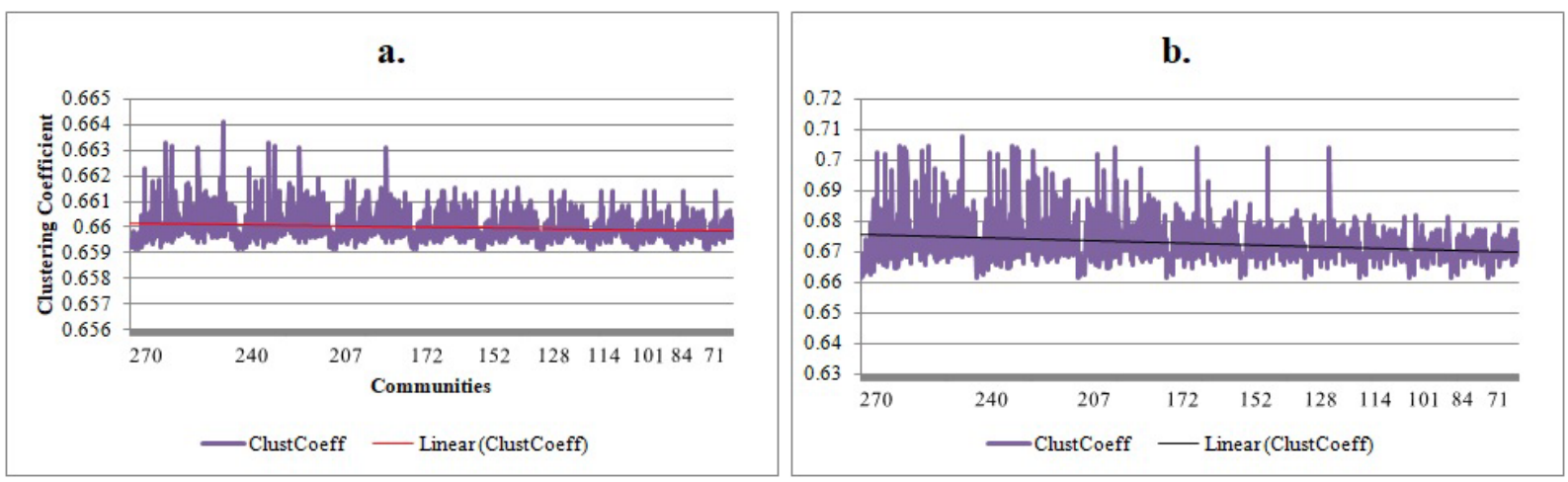

FIG. 8.2. Number of communities VS Clustering Coefficient (a) Amazon (b)AstroPh
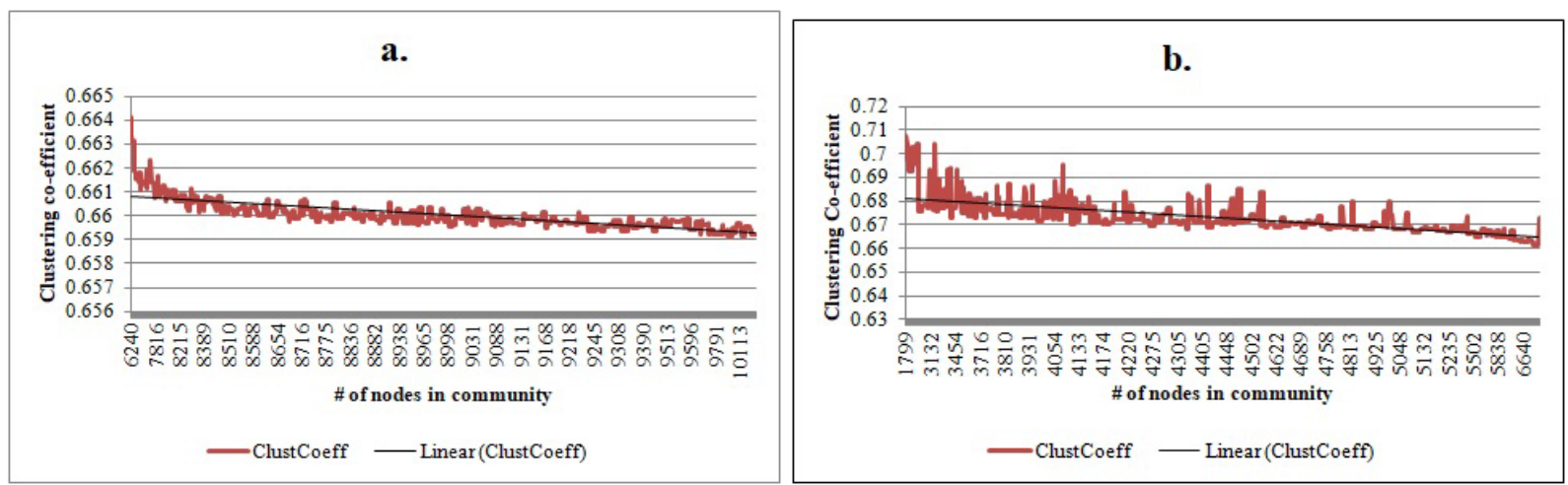

FIG. 8.3. Number of nodes in the community VS Clustering Coefficient (a) Amazon (b)AstroPh

the Table 8.1. OSLOM [16], DEMON [4], Big Clam [39], nise-sph-fppr and nise-grc-fppr from [35].

As it can be clearly seen in the Table 8.1 the proposed P4S algorithm with seed expansion algorithm works faster than the other algorithms compared. Since it is parallel execution, the algorithm finishes its execution faster than the other algorithms compared.

The details in the Table 8.2 depict the coverage (\%) of each algorithm. In this case the proposed algorithm doesnt gives $100 \%$ as nise-sph-fprr and nise-grc-fppr [35]. When the number of communities is considered the proposed $\mathrm{P} 4 \mathrm{~S}$ method gives better result than the algorithms compared.

9. Conclusion. The proposed $\mathrm{P} 4 \mathrm{~S}$ method has been implemented and tested in this article. The work gives encouraging result and it could produce better communities in less time. The P4S selects very good seeds and because of the seeds he communities can be expanded from the seeds easily. Since the seeds have a good density of nodes around it, the expansion will be faster when the process starts and it drags a little to complete. The generated communities from this method prove the seeds selected are excellent. The goodness metric of the communities selected has been tested with the density metrics. The density test proves that the communities generated are also good. The communities detected has also been tested with the clustering coefficient and proved to be good after the detection of community. The trend line shows that larger the seed set higher the density will be. The runtime of the algorithm was also calculated and it shows a very good improvement. Finally, the coverage of the algorithm was tested and the P4S covers more than $95 \%$ in most of the iterations. When considering the number of communities with the coverage $\%$ the result $\mathrm{P} 4 \mathrm{~S}$ gave is better. The algorithm still needs many improvements with respect to the centrality measures selection, range of threshold values and seed expansion approach. 

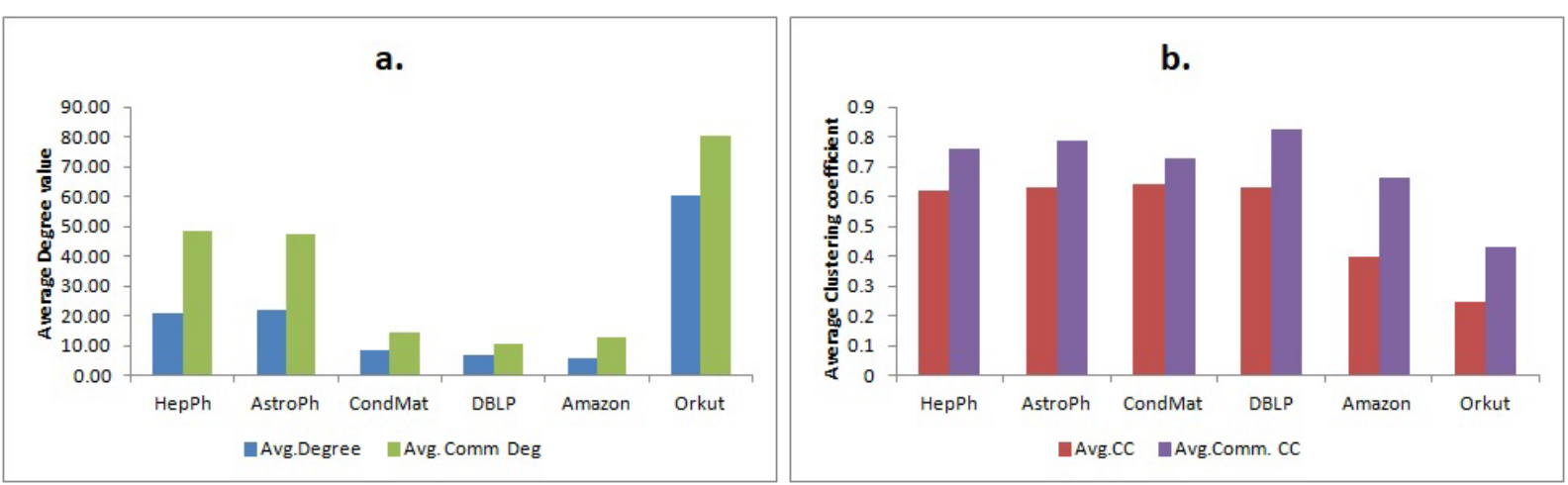

FIG. 8.4. (a) Comparison of average clustering coefficient of nodes before and after community detection. (b) Comparison of average degree of nodes before and after community detection.

TABLE 8.1

Running times of different methods on our test networks in (Minutes)

\begin{tabular}{|l|l|l|l|l|l|l|}
\hline Graph & oslom & demon & bigclam & nise-sph-fppr & nise-grc-fppr & P4S \\
\hline HepPh & 19.26 & 0.45 & 11.383 & 0.36 & 2.8 & 0.3 \\
\hline AstroPh & 38.05 & 0.7 & 48.016 & 0.6 & 2.43 & 0.483 \\
\hline CondMat & 20.65 & 0.83 & 7.35 & 0.6 & 1.23 & 0.45 \\
\hline DBLP & 350 & 233 & 433 & 18.33 & 29.733 & 12.6 \\
\hline Amazon & 175 & 115 & 85 & 37.6 & 42.716 & 20.3 \\
\hline Orkut & N/A & N/A & 4199 & 43.916 & 236 & 31.83 \\
\hline
\end{tabular}

TABLE 8.2

Returned number of communities and graph coverage of each algorithm

\begin{tabular}{|l|l|l|l|l|l|l|l|}
\hline Graph & & oslom & demon & bigclam & nise-sph-fppr & nise-grc-fppr & P4S \\
\hline \multirow{2}{*}{ HepPh } & Coverage (\%) & 100 & 88.3 & 84.37 & 100 & 100 & 94.83 \\
& no.of.clusters & 608 & 5,147 & 100 & 99 & 90 & 270 \\
\hline \multirow{2}{*}{ AstroPh } & Coverage (\%) & 100 & 94.15 & 91.11 & 100 & 100 & 95.4 \\
& no.of.clusters & 1,241 & 8,259 & 200 & 212 & 246 & 270 \\
\hline \multirow{2}{*}{ CondMat } & Coverage (\%) & 100 & 91.16 & 99.96 & 100 & 100 & 98.5 \\
& no.of.clusters & 1,534 & 10,474 & 200 & 201 & 249 & 270 \\
\hline \multirow{2}{*}{ DBLP } & Coverage (\%) & 100 & 84.89 & 100 & 100 & 100 & 97.4 \\
& no.of.clusters & 17,519 & 174,560 & 25,000 & 26,503 & 18,477 & 270 \\
\hline \multirow{2}{*}{ Amazon } & Coverage (\%) & 100 & 79.16 & 100 & 100 & 100 & 96.71 \\
& no.of.clusters & 17,082 & 105,685 & 25,000 & 27,763 & 20,036 & 270 \\
\hline \multirow{2}{*}{ Orkut } & Coverage (\%) & N/A & N/A & 82.13 & 99.99 & 99.99 & 98.5 \\
& no.of.clusters & N/A & N/A & 25,000 & 25,204 & 32,622 & 270 \\
\hline
\end{tabular}




\section{REFERENCES}

[1] R. Belfin, G. E., and P. Bródka. Overlapping community detection using superior seed set selection in social networks. Computers and Electrical Engineering, 2018.

[2] P. Bonacich. Power and Centrality: A Family of Measures. American Journal of Sociology, 1987.

[3] T. Chakraborty, S. Srinivasan, N. Ganguly, A. Mukherjee, and S. Bhowmick. Permanence and Community Structure in Complex Networks. 11(2), 2016.

[4] M. Coscia, G. Rossetti, F. Giannotti, and D. Pedreschi. DEMON: a Local-First Discovery Method for Overlapping Communities. 2012.

[5] J. Dean and S. Ghemawat. MapReduce: Simplified Data Processing on Large Clusters. Communications of the ACM, 2008.

[6] S. Dhamal, P. K. J., and Y. Narahari. Information Diffusion in Social Networks in Two Phases. IEEE Transactions on Network Science and Engineering, 4697(c):1-1, 2016

[7] F. Erlandsson, P. Brdóka, and A. Borg. Seed selection for information cascade in multilayer networks. Studies in Computational Intelligence, 689:426-436, 2018.

[8] W. Fan, J. Xu, X. Luo, Y. Wu, W. Yu, and R. Xu. GRAPE: Conducting Parallel Graph Computations without Developing Parallel Algorithms. pages 30-41.

[9] L. C. Freeman. Centrality in Social Networks. Social Networks, 1978.

[10] L. C. Freeman. Centrality in social networks conceptual clarification. Social Networks, 1978.

[11] J. Han, W. Li, Z. Su, L. Zhao, and W. Deng. Community detection by label propagation with compression of flow. 2016.

[12] L. G. S. Jeub, M. W. Mahoney, P. J. Mucha, and M. A. Porter. A Local Perspective on Community Structure in Multilayer Networks. volume 5, pages 144-163, 2015.

[13] R. Kanawati. Seed-Centric Approaches for Community Seed-Centric Algorithms : A Classification Study. pages 197-208, 2014.

[14] R. Kanawati. YASCA: An Ensemble-Based Approach for Community Detection in Complex Networks. Algorithmica, 76(4):657-666, 2014.

[15] A. Lancichinetti and S. Fortunato. Community detection algorithms: A comparative analysis. Physical Review E - Statistical, Nonlinear, and Soft Matter Physics, 80(5):1-11, 2009.

[16] A. Lancichinetti, F. Radicchi, J. J. Ramasco, and S. Fortunato. Finding statistically significant communities in networks. PLoS ONE, 6(4), 2011.

[17] S. Large and N. Dataset. Stanford Large Network Dataset Collection, 2013.

[18] Y. Li, Y. Wang, J. Chen, L. Jiao, and R. Shang. Overlapping community detection through an improved multi-objective quantum-behaved particle swarm optimization. Journal of Heuristics, 21(4):549-575, 2015.

[19] Y. Low, J. Gonzalez, A. Kyrola, D. Bickson, C. Guestrin, and J. M. Hellerstein. GraphLab: A New Framework for Parallel Machine Learning. The 26th Conference on Uncertainty in Artificial Intelligence (UAI 2010), 2010.

[20] G. Malewicz, M. H. Austern, A. J. Bik, J. C. Dehnert, I. Horn, N. Leiser, and G. Czajkowski. Pregel: a system for large-scale graph processing. Proceedings of the 2010 international conference on Management of data - SIGMOD '10, 2010.

[21] R. Michalski, T. Kajdanowicz, P. Bródka, and P. Kazienko. Seed selection for spread of influence in social networks: Temporal vs. static approach. New Generation Computing, 32(3-4):213-235, 2014.

[22] C. H. Mu, J. Xie, Y. Liu, F. Chen, Y. Liu, and L. C. Jiao. Memetic algorithm with simulated annealing strategy and tightness greedy optimization for community detection in networks. Applied Soft Computing Journal, 34:485-501, 2015.

[23] S. Salihoglu and J. Widom. GPS : A Graph Processing System. SSDBM Proceedings of the 25th International Conference on Scientific and Statistical Database Management, 2013.

[24] A. Sol, S. G. e-Ribalta, Manlio De Domenico, and A. A. Omez. Random walk centrality in interconnected multilayer networks. Physica D: Nonlinear Phenomena, 323-324:73-79, 2016.

[25] L. Solá, M. Romance, R. Criado, J. Flores, A. García del Amo, and S. Boccaletti. Eigenvector centrality of nodes in multiplex networks. Chaos, 23(3):1-11, 2013.

[26] G. Song, Y. Li, X. Chen, X. He, and J. Tang. Influential Node Tracking on Dynamic Social Network: An Interchange Greedy Approach. IEEE Transactions on Knowledge and Data Engineering, 29(2):359-372, 2017.

[27] A. Srivastava, C. Chelmis, and V. Prasanna. Social influence computation and maximization in signed networks with competing cascades. Proceedings of the 2015 IEEE/ACM International Conference on Advances in Social Networks Analysis and Mining, ASONAM 2015, pages 41-48, 2015.

[28] V. Tejaswi, P. V. Bindu, and P. S. Thilagam. Diffusion models and approaches for influence maximization in social networks. In 2016 International Conference on Advances in Computing, Communications and Informatics, ICACCI 2016, pages 1345-1351, 2016.

[29] L. G. . V. . Valiant. A Bridging Model for Parallel Computation. Communications of the ACM, 1990.

[30] G. Wang, W. Xie, A. Demers, and J. Gehrke. Asynchronous Large-Scale Graph Processing Made Easy. Cidr, 2013.

[31] M. Wang, C. Wang, J. X. Yu, and J. Zhang. Community Detection in Social Networks : An In-depth Benchmarking Study with a Procedure-Oriented Framework. Proceedings of the VLDB Endowment, pages 998-1009, 2015.

[32] Y. Wang, B. Zhang, A. V. Vasilakos, and J. Ma. PRDiscount: A Heuristic scheme of initial seeds selection for diffusion maximization in social networks. In Lecture Notes in Computer Science (including subseries Lecture Notes in Artificial Intelligence and Lecture Notes in Bioinformatics), volume 8588 LNCS, pages 149-161, 2014.

[33] D. J. Watts and S. H. Strogatz. Collective dynamics of small-world' networks. Nature, 393(6684):440-442, 1998.

[34] M. Weskida. Evolutionary Algorithm for Seed Selection in Social Influence Process. pages 1189-1196, 2016.

[35] J. J. Whang, D. F. Gleich, and I. S. Dhillon. Overlapping Community Detection Using Neighborhood-Inflated Seed Expansion. IEEE Transactions on Knowledge and Data Engineering, 28(5):1272-1284, 2016. 
[36] J. J. Whang, P. Rai, and I. S. Dhillon. Stochastic blockmodel with cluster overlap, relevance selection, and similarity-based smoothing. Proceedings - IEEE International Conference on Data Mining, ICDM, pages 817-826, 2013.

[37] R. S. Xin, J. E. Gonzalez, M. J. Franklin, and I. Stoica. GraphX. In First International Workshop on Graph Data Management Experiences and Systems - GRADES '13, pages 1-6, 2013.

[38] D. Yan, J. Cheng, Y. Lu, and W. Ng. Blogel - a block-centric framework for distributed computation on real-world graphs. Proceedings of the VLDB Endowment, 7(14):1981-1992, 2014.

[39] J. Yang and J. Leskovec. Overlapping community detection at scale: A Nonnegative Matrix Factorization Approach. Sixth ACM international conference on Web search and data mining, page 587, 2013.

[40] J. U. N. Zhang, F. Xia, S. Member, and Z. Ning. A Hybrid Mechanism for Innovation Diffusion in Social Networks. $4,2016$.

Edited by: Rajkumar Rajasekaran

Received: Jul 30, 2018

Accepted: Dec 14, 2018 